\title{
CONTINUOUS FAMILIES OF ISOPHASAL SCATTERING MANIFOLDS
}

\author{
Carolyn Gordon and Peter Perry
}

To the Memory of Robert Brooks

\begin{abstract}
We construct continuous families of Riemannian metrics on $\mathbf{R}^{n}$ for $n \geq 8$ with the same scattering phase and resonances. The metrics are compactly supported perturbations, with arbitrarily small support, of the Euclidean metric and have infinitely many scattering resonances.
\end{abstract}

\section{Introduction}

Inverse spectral geometry for compact Riemannian manifolds is the study of which geometric properties of the manifold are determined by the eigenvalues of the Laplacian. For non-compact Riemannian manifolds, there may be only finitely many $L^{2}$ eigenvalues of the Laplacian (or no $L^{2}$-eigenvalues at all) but there are several possible analogues of spectral data for which one can pose a similar inverse problem. In many noncompact settings, one can define scattering resonances, which serve as discrete data analogous to the eigenvalues in the compact setting. Two metrics are said to be isopolar if they have the same scattering resonances, with mulitiplicities. In the setting we will consider, one can further define a notion of scattering phase, a function roughly analogous to the counting function for eigenvalues in the compact problem. The condition that two metrics be isophasal, i.e. that they have the same scattering phase, is stronger than isopolarity.

In the compact setting, there is a well-developed literature of both positive and negative inverse spectral results. In particular, a vast array of isospectral manifolds reveals many geometric and topological invariants that are not spectrally determined. There are two known systematic methods for constructing isospectral manifolds; most of the known examples were either constructed by or can be explained by one of these two methods. (i) Representation theoretic techniques, most notably the Sunada method, have been used very extensively to construct isospectral manifolds with a common Riemannian cover. (There is one exception: C. Sutton used representation theoretic techniques to construct a pair of isospectral simply-connected manifolds; in particular they do not have a common Riemannian cover.) (ii) A technique involving torus actions and Riemannian submersions, developed over the past decade, has given rise to many examples of isospectral metrics with different local geometry including, among others, the first examples of isospectral closed manifolds [8], the first examples

Received by the editors April 18, 2005.

Key words and phrases. Geometric scattering, scattering manifold, isophasal manifold.

Gordon supported in part by NSF grants DMS-0072534 and DMS-0306752.

Perry supported in part by NSF grants DMS-9707051 and DMS-0100829. 
of isospectral simply-connected manifolds [22], families of isospectral left-invariant metrics on the classical compact Lie groups [23], [19], and continuous families of isospectral metrics on spheres and balls [9], [24].

Much less is known in the scattering setting. All known examples to date have come about by adapting the Sunada technique from the compact to the non-compact setting. This has resulted in many interesting examples: finite-area Riemann surfaces (both isopolar and isophasal-see Bérard [1] and Zelditch [29], [30]), Riemann surfaces of infinite area (isopolar and isophasal-see Guillopé-Zworski [11] and BrooksDavidovich [3]), three-dimensional Schottky manifolds (isopolar-see Brooks-GornetPerry [4]), and surfaces with non-trivial topology that are isometric to Euclidean space outside a compact set (isopolar and isophasal-see Brooks-Perry [5]). However, the Sunada method has the same limitations as in the compact setting. In particular, the manifolds in each of these examples have a common finite covering. Moreover, in each case there are only finite sets of isopolar (or isophasal) manifolds as opposed to continuous deformations.

In this article, we show that the method of torus actions and Riemannian submersions can also be adapted to the noncompact setting. We will focus here on a particularly attractive setting, namely the inverse scattering problem on Euclidean $\mathbf{R}^{n}$ with compactly supported perturbations of the Euclidean metric.

We will prove:

Theorem 1.1. For every $n \geq 8$, there exist continuous families of isophasal, nonisometric Riemannian metrics on $\mathbf{R}^{n}$ which are Euclidean outside of a compact set of arbitrarily small volume. There also exist pairs of such metrics on $\mathbf{R}^{6}$.

Remark 1.2. Letting $m=n-4$, the parameter space for the continuous families of isophasal metrics on $\mathbf{R}^{n}$ that we will construct has dimension

$$
d \geq \frac{m(m-1)}{2}-\left[\frac{m}{2}\right]\left(\left[\frac{m}{2}\right]+2\right)>1
$$

if $n=9$ or $n \geq 11$. (If $n=8$ or $n=10$, the parameter space has dimension at least $1)$.

In addition to proving the existence of the continuous families, we will give an explicit example of a triple of isophasal metrics on $\mathbf{R}^{12}$. We will see that these metrics have very different geometry. Indeed their isometry groups have different dimension and structure.

To our knowledge, the isophasal metrics of Theorem 1.1 differ from the other known examples of isophasal or isopolar metrics in the following ways:

- They are the first continuous families of isophasal or isopolar metrics;

- They are the first simply-connected examples, in fact the first for which the manifolds do not share a common Riemannian cover;

- They are the first isophasal or isopolar compact metric perturbations of the Euclidean metric on $\mathbf{R}^{n}$.

We feel that this initial generalization of the torus method to the noncompact setting will open a new line of investigation. Already we are adapting the method to address the construction both of isophasal potentials for the Schrodinger operator and of isophasal obstacles. These constructions will be addressed in a later paper. 
The metrics that we construct are extension to the full Euclidean space of isospectral families of metrics on balls and spheres constructed in Gordon [9] and Schueth [24]. In particular, the continuous families of isosphasal metrics that we construct exhibit the strong property that their restrictions to any ball or sphere centered at the origin are isospectral. These examples illustrate that isophasality and isopolarity are natural extensions of the notion of isospectrality.

We now review the notion of scattering resonances in our setting. For the class of manifolds that we consider here, the Laplacian has purely continuous spectrum in $[0, \infty)$ and no $L^{2}$-eigenvalues. Thus, the resolvent of the Laplacian is an analytic function $R(z)=\left(\Delta_{X}-z\right)^{-1}$ on $\mathbf{C} \backslash[0, \infty)$; the resolvent admits a meromorphic continuation to a double covering of the complex plane if $n$ is odd, and a logarithmic covering of the complex plane if $n$ is even (this result follows, for example, from the "black box scattering" formalism introduced by Sjöstrand and Zworski in [26]). Resolvent resonances are poles of the meromorphically continued resolvent. In the literature they are also referred to simply as resonances. Because they are defined by a meromorphic continuation, resonances are less easily studied than eigenvalues, but understanding their geometric content is an important problem. For the case considered here, the resolvent resonances are identical to the poles of the meromorphically continued scattering operator, which are called scattering resonances. We define and discuss the scattering operator, scattering resonances with multiplicities, and the notion of scattering phase in $\S 2$ of what follows.

One might worry that the examples constructed have trivial scattering (e.g., have no scattering poles). We show, however, that the isosphasal metrics can always be chosen to have infinitely many resonances. For metric scattering on $\mathbf{R}^{n}$, Sá Barreto and Tang [21] ( $n$ odd) and Tang [28] ( $n$ even) proved the existence of infinitely many resonances so long as the second relative heat invariant $a_{2}$ is non-vanishing. They also gave various geometric hypotheses which guarantee the non-vanishing of $a_{2}$ : one of these is that the given metric is not flat but is a compactly supported perturbation of the Euclidean metric that is close in the $\mathcal{C}^{k}$ topology to the Euclidean metric for sufficiently large $k$ (Theorem 1.3 of [21] and Theorem 1.1 of [28]) ${ }^{1}$. In our examples, it is easily verified that the metrics are not flat, and it is easy to construct examples where the metrics are arbitrarily close in the $\mathcal{C}^{k}$ sense to the Euclidean metric for $k$ large and fixed ${ }^{2}$. We can actually remove the assumption that our metrics are $\mathcal{C}^{k}$ close to the Euclidean metric by computing the $a_{2}$ heat invariant directly, at the cost of imposing a genericity assumption ${ }^{3}$ on the space of metrics; we carry out this computation in Section 5 for the metrics on $\mathbf{R}^{n}$ with $n \geq 9$.

\footnotetext{
${ }^{1}$ Both of these papers rely on a result of Kuwabara [17] which states that, given a flat metric $\gamma$ on a compact manifold $X$, there is a neighborhood $U$ of $\gamma$ in the $\mathcal{C}^{\infty}$ topology so that if $a_{2}(g)=0$ and $g \in U$, then $g$ is flat. In fact, a close examination of [17] shows that it is sufficient for $g$ and $\gamma$ to be close in the $\mathcal{C}^{k}$ topology for $k$ sufficiently large: see Theorem A' of section 6 in [17]. For the connection between Kuwabara's result on compact manifolds and the result on metric perturbations of Euclidean $\mathbf{R}^{n}$, see the proof of Theorem 1.3 of [21] which uses finite propagation speed for solutions of the wave equation.

${ }^{2}$ As explained in Section 4, the metrics depend on a skew-symmetric bilinear form and a $\mathcal{C}_{0}^{\infty}\left(\mathbf{R}^{n}\right)$ function $\varphi$ which defines the support of the perturbation. One takes the function $\varphi$ sufficiently small in $\mathcal{C}^{k}$-sense.

${ }^{3}$ The genericity condition we impose is merely a genericity condition on the choice of cut-off function $\varphi$ which defines the metric; see Definition 4.3 in what follows.
} 
The plan of this paper is as follows. In $\S 2$, we discuss basics of scattering theory for asymptotically Euclidean manifolds. In $\S 3$, we develop a technique, based on the method of torus actions, for constructing manifolds with the same scattering phase (see Theorem 3.7). In $\S 4$ we apply the technique of the previous section to show that the metrics on balls and spheres constructed in [9] and [24] extend to isophasal metrics on $\mathbf{R}^{n}$, thus proving Theorem 1.1. We give full details for the examples in dimension $n \geq 9$, based on the examples in [9]). The lower-dimensional examples are given by extensions of metrics constructed in [24]. Since the methods of $\S 3$ apply in exactly the same way to these examples, we do not include the details here. Finally, in $\S 5$, we carry out the explicit computation that the $a_{2}$ heat invariant is generically nonvanishing, again restricting our attention to the examples in dimension 9 and above.

\section{Metric scattering on $\mathbf{R}^{n}$}

In this section we review scattering theory for manifolds $X=\left(\mathbf{R}^{n}, g\right)$ where $g$ is a compactly supported metric perturbation of the Euclidean metric: see [14] and [15] for an expository treatment that includes the case considered here. Letting $\Delta_{X}$ be the positive Laplace-Beltrami operator on $X$, it follows from the classical Rellich uniqueness theorem that $\Delta_{X}$ has no $L^{2}$-eigenvalues, and it is easy to prove that $\Delta_{X}$ has purely absolutely continuous spectrum in $[0, \infty)$. Thus the resolvent operator $\widetilde{R}(z)=\left(\Delta_{X}-z\right)^{-1}$, considered as a mapping from $L^{2}(X)$ to itself, is an operatorvalued analytic function of $z$ in $\mathbf{C} \backslash[0, \infty)$. It can be shown that the mapping $R(\lambda)=$ $\widetilde{R}\left(\lambda^{2}\right)$, initially defined on the half-plane $\Im(\lambda)>0$ and viewed as a map from $\mathcal{C}_{0}^{\infty}\left(\mathbf{R}^{n}\right)$ to $\mathcal{C}^{\infty}\left(\mathbf{R}^{n}\right)$, admits a meromorphic continuation to the complex $\lambda$-plane if $n$ is odd, and to the logarithmic plane if $n$ is even. At any pole $\zeta$, the resolvent admits a Laurent expansion with finite polar part of the form

$$
\sum_{j=1}^{N_{\zeta}} \frac{A_{j}}{\lambda-\zeta}
$$

where the $A_{j}$ are finite-rank operators from $\mathcal{C}_{0}^{\infty}\left(\mathbf{R}^{n}\right)$ to $\mathcal{C}^{\infty}\left(\mathbf{R}^{n}\right)$. The multiplicity of the pole $\zeta$ is defined as $\operatorname{dim}\left(\oplus_{j}\left(\operatorname{Ran} A_{j}\right)\right)$.

To define the scattering phase, we first recall that the absolutely continuous spectrum is parameterized by scattering solutions to the eigenvalue equation

$$
\left(\Delta_{X}-\lambda^{2}\right) u=0
$$

which are easily described. In what follows, write $x \in \mathbf{R}^{n} \backslash\{0\}$ as $x=r \omega$ where $r>0$ and $\omega \in S^{n-1}$.

Proposition 2.1. Fix $f_{-} \in \mathcal{C}^{\infty}\left(S^{n-1}\right)$ and $\lambda>0$. There exists a unique $\mathcal{C}^{\infty}$ solution of the equation

$$
\left(\Delta_{X}-\lambda^{2}\right) u=0
$$

having the asymptotic form

$$
u(r \omega)=r^{(1-n) / 2} e^{i \lambda r} f_{+}(\omega)+r^{(1-n) / 2} e^{-i \lambda r} f_{-}(\omega)+\mathcal{O}\left(r^{-(n+1) / 2}\right)
$$

as $r \rightarrow \infty$. In particular, the function $f_{+} \in \mathcal{C}^{\infty}\left(S^{n-1}\right)$ is uniquely determined. 
For a proof see [14].

The Proposition implies that the mapping $f_{-} \mapsto f_{+}$is a well-defined mapping from $\mathcal{C}^{\infty}\left(S^{n-1}\right)$ to itself. We denote this map, the absolute scattering matrix for $X$, by $S(\lambda)$. From the definition, it is clear that $S(\lambda)$ is a linear mapping, and that $S(\lambda)^{-1}=S(-\lambda)$ for real $\lambda \neq 0$. In the case of $X_{0}=\left(\mathbf{R}^{n}, g_{0}\right)$ (where $g_{0}$ is the Euclidean metric on $\mathbf{R}^{n}$ ), we have the explicit formula

$$
u(x)=\int_{S^{2}} \exp (-i \lambda x \cdot \omega) f_{-}(\omega) d \omega
$$

and a stationary phase calculation shows that the absolute scattering matrix $S_{0}(\lambda)$ is given by

$$
\left(S_{0}(\lambda) \varphi\right)(\omega)=i^{n-1} \varphi(-\omega) .
$$

Since $X$ is a compactly supported metric perturbation of $X_{0}$, it is not surprising that the 'relative scattering matrix'

$$
S_{r}(\lambda)=S(\lambda) S_{0}(\lambda)^{-1}
$$

has especially nice properties (see, for example, $\S 5.2$ of [15]):

Proposition 2.2. For real $\lambda \neq 0$, the relative scattering matrix $S_{r}(\lambda)$ extends to a unitary operator from $L^{2}\left(S^{n-1}\right)$ to itself. Moreover

$$
S_{r}(\lambda)=I+T(\lambda)
$$

where $T(\lambda)$ is an integral operator with integral kernel belonging to $\mathcal{C}^{\infty}\left(S^{n-1} \times S^{n-1}\right)$.

In particular, $T(\lambda)$ extends to a trace-class operator on $L^{2}\left(S^{n-1}\right)$, so that the operator determinant

$$
\operatorname{det} S_{r}(\lambda)=\operatorname{det}(I+T(\lambda))
$$

is well-defined (see, for example, [25] for a discussion of operator determinants). Since $S_{r}(\lambda)$ is unitary, it follows that $\operatorname{det} S_{r}(\lambda)$ has modulus one. We note for use later that if $A$ is a trace-class operator on a Hilbert space $\mathcal{H}$ and $B$ is a boundedly invertible linear operator on $\mathcal{H}$, the equality

$$
\operatorname{det}(I+A)=\operatorname{det}\left(I+B A B^{-1}\right)
$$

holds.

It can be shown that the determinant $\operatorname{det}\left(S_{r}(\lambda)\right)$ extends to a meromorphic function on the complex plane ( $n$ odd) or the logarithmic plane ( $n$ even) whose poles coincide, including multiplicity, with the resolvent resonances.

The real-valued function

$$
\sigma(\lambda)=\frac{1}{2 \pi i} \log \operatorname{det}\left(S_{r}(\lambda)\right)
$$

on $(0, \infty)$ is called the scattering phase and behaves in many respects analogously to the counting function for eigenvalues on a compact manifold. For example, Christiansen [7] has shown that the scattering phase for a class of scattering manifolds including those considered here obeys the asymptotic law

$$
\sigma(\lambda)=-c_{n} \operatorname{sc}-\operatorname{vol}(X, g) \lambda^{n}+\mathcal{O}\left(\lambda^{n-1}\right)
$$


as $\lambda \rightarrow \infty$, where, for the case of compactly supported metric perturbations of the Euclidean metric,

$$
\operatorname{sc}-\operatorname{vol}(X, g)=\lim _{\varepsilon \downarrow 0}\left(\int_{X_{\varepsilon}} d g-\frac{1}{n} \operatorname{vol}\left(S^{n-1}\right) \varepsilon^{-n}-c_{n-1} \varepsilon^{n-1}-\cdots-c_{0} \log \varepsilon\right)
$$

(previously, Robert [20] obtained asymptotics of the scattering phase for potential and metric perturbations of the Euclidean metric on $\mathbf{R}^{n}$ ). The constant $c_{n}$ is the same constant that appears in Weyl's law for the counting function of eigenvalues. The constants $c_{k}$ are chosen to make the limit finite, and $X_{\varepsilon}$ is the compact set in $\mathbf{R}^{n}$ with $|x| \leq \varepsilon^{-1}$ (equivalently, sc-vol $(X, g)$ is the Hadamard finite part of $\operatorname{vol}_{g}\left(X_{\varepsilon}\right)$ as $\varepsilon \downarrow 0)$. Note that $\mathrm{sc}-\operatorname{vol}(X, g)$ may be positive, negative, or zero, depending on $g$.

In the examples we will construct, the volume forms for $g$ and $g_{0}$ are identical, so $\operatorname{sc}-\operatorname{vol}(X, g)=0$. Thus our examples have "weak scattering" in the sense that $\sigma(\lambda)=\mathcal{O}\left(\lambda^{n-1}\right)$. In a subsequent paper, we will show how to construct continuous families of isospectral manifolds with $\operatorname{sc}-\operatorname{vol}(X, g) \neq 0$.

\section{Technique for constructing isosphasal manifolds}

Before presenting the method we will use for constructing isosphasal metrics, we review basic properties of group actions, in particular, torus actions. Given an action of a compact Lie group $G$ on a manifold $M$, the principal orbits are the orbits with minimal isotropy. The union of the principal orbits is an open dense subset $M^{\prime}$ of $M$. (See [2], Theorem 3.1.) There exists a subgroup $H$ of $G$ such the isotropy group of every element of $M^{\prime}$ is conjugate to $H$. Moreover, the isotropy group of an arbitrary element of $M$ contains a subgroup conjugate to $H$. In case $G$ is a torus, it follows that the isotropy group of every element contains $H$ itself. In particular, if a torus action is effective, then $H$ is trivial and so the action on the principal orbits is free. Thus $M^{\prime}$ is a principal $G$-bundle.

Notation 3.1. Suppose a torus $T$ acts smoothly on a connected manifold $M$. For each character $\alpha: T \rightarrow S^{1}$ (where $S^{1}$ is the unit circle in $\mathbf{C}$ ), write

$$
\mathcal{H}_{\alpha}=\left\{f \in C^{\infty}(M): f(z \cdot x)=\alpha(z) f(x) \text { for all } x \in M, z \in T\right\} .
$$

For $K$ a subtorus of $T$ of codimension at most one, let $\mathcal{C}^{\infty}(M)^{K}$ denote the space of $K$-invariant smooth functions on $M$. Then

$$
\mathcal{C}^{\infty}(M)^{K}=\oplus_{\alpha: K \subset \operatorname{ker}(\alpha)} \mathcal{H}_{\alpha}
$$

Thus by Fourier analysis, we may decompose $\mathcal{C}^{\infty}(M)$ as

$$
\mathcal{C}^{\infty}(M)=\mathcal{C}^{\infty}(M)^{T} \oplus\left(\oplus_{K}\left(\mathcal{C}{ }^{\infty}(M)^{K} \ominus \mathcal{C}^{\infty}(M)^{T}\right)\right)
$$

where $K$ varies over all subtori of codimension one.

Notation 3.2. If $(M, g)$ is a complete Riemannian manifold, we denote by $\mathcal{C}_{2}^{k}(M, g)$ the set of $\mathcal{C}^{\infty}$ functions $u$ for which $u, \nabla u, \cdots, \nabla^{k} u$ belong to $L^{2}(M, g)$. We denote by $H^{k}(M, g)$ the completion of $\mathcal{C}_{2}^{k}(M, g)$ in the norm

$$
\|u\|_{H^{k}}=\left(\sum_{j=0}^{k}\left\|\nabla^{j} u\right\|_{L^{2}(M, g)}^{2}\right)^{1 / 2} .
$$


We denote by $H_{0}^{1}(M, g)$ the completion of $\mathcal{C}_{0}^{\infty}(M)$ with respect to the inner product

$$
(\varphi, \psi)_{H^{1}(M, g)}=\int_{M} \varphi \bar{\psi} d v_{g}+\int \nabla \varphi \cdot \overline{\nabla \psi} d v_{g}
$$

Since, by a theorem of Chernoff [6], $C_{0}^{\infty}(M)$ is a domain of essential self-adjointness for $\Delta_{g}$, it is also dense in the form domain of $\Delta_{g}$. We denote by $H_{0}^{k}(M, g)$ the completion of $\mathcal{C}_{0}^{\infty}(M)$ in the norm $\|u\|_{H^{k}(M, g)}=\left\|\left(\Delta_{g}+1\right)^{k / 2} u\right\|$. The domain of $\Delta_{g}$ is $H_{0}^{2}(M, g)$, and similarly the domain of $\Delta_{g}^{k}$ is $H_{0}^{2 k}(M, g)$ [6]. If $(M, g)$ has positive injectivity radius and the functions $\nabla^{j}$ Ric, $j=0, \cdots, k-2$ are bounded (here Ric is the Ricci curvature of $(M, g))$, the spaces $H_{0}^{k}(M, g)$ coincide with the spaces $H^{k}(M, g)$ (see, for example Hebey [13], Proposition 3.2). Under these hypotheses, the inclusion

$$
H_{0}^{k}(M, g) \subset \mathcal{C}^{\ell}(M)
$$

holds provided $k>n / 2+\ell$ (see, for example, [13], Theorem 3.4).

The following proposition adapts ideas of Gordon [9] and Schueth [24] to the noncompact setting.

Proposition 3.3. Let $\left(M_{1}, g_{1}\right)$ and $\left(M_{2}, g_{2}\right)$ be connected, complete Riemannian manifolds with positive injectivity radius and $\nabla^{k} \mathrm{Ric}_{i}$ bounded for $i=1,2$ and all nonnegative integers $k$. Let $T$ be a torus. Suppose $T$ acts effectively by isometries on $\left(M_{1}, g_{1}\right)$ and $\left(M_{2}, g_{2}\right)$ and that the action of $T$ on the principal orbits is free. Let $M_{i}^{\prime}$ be the union of all principal orbits in $M_{i}$, so $M_{i}^{\prime}$ is an open, dense submanifold of $M_{i}$ and a principal $T$-bundle, $i=1,2$. For each subtorus $K$ of $T$ of codimension at most one, suppose that there exists a T-equivariant volume-preserving diffeomorphism $F_{K}: M_{1} \rightarrow M_{2}$ that induces an isometry $\bar{F}_{K}$ between the induced metrics on the quotient manifolds $K \backslash M_{1}^{\prime}$ and $K \backslash M_{2}^{\prime}$. With respect to the Fourier decompositions of $\mathcal{C}^{\infty}\left(M_{1}\right)$ and $\mathcal{C}^{\infty}\left(M_{2}\right)$ given in Notation 3.1, let

$$
Q=F_{T}^{*} \oplus\left(\oplus_{K} F_{K}^{*}\right),
$$

initially defined as a map from $\mathcal{C}_{0}^{\infty}\left(M_{2}^{\prime}\right)$ to $\mathcal{C}_{0}^{\infty}\left(M_{1}^{\prime}\right)$. Then $Q$ extends to a map from $\mathcal{C}^{\infty}\left(M_{2}\right)$ to $\mathcal{C}^{\infty}\left(M_{1}\right)$ with the property that

$$
\Delta_{1} \circ Q=Q \circ \Delta_{2},
$$

where $\Delta_{i}$ is the Laplace operator on $\mathcal{C}^{\infty}\left(M_{i}\right)$.

Remark 3.4. Proposition 3.3 as stated here differs somewhat from the original statements in [9] and [24]. There the manifolds $M_{i}$ were assumed to be compact and the conclusion was that they were isospectral. However, the construction of the intertwining operator $Q$ did not use the assumption that the manifolds were compact. A second difference between the statement here and that in [9] is that a hypothesis involving preservation by the diffeomorphisms $F_{K}$ of the mean curvature of the fibers has been replaced by the condition that these diffeomorphisms be volume-preserving. Dorothee Schueth made this simplifying change in her version [24] of the proposition, observing that the former and latter conditions are equivalent.

Remark 3.5. In [24], Scheuth actually shows that the map $Q$ is an isometry between the spaces $H^{1}\left(M_{1}, g_{1}\right)$ and $H^{1}\left(M_{2}, g_{2}\right)$, assuming that $M_{1}$ and $M_{2}$ are compact manifolds, possibly with boundary. Her proof goes through with no essential change to 
show that the map $Q$ is also an isometry between $H^{1}\left(M_{1}, g_{1}\right)$ and $H^{1}\left(M_{2}, g_{2}\right)$ when $\left(M_{i}, g_{i}\right)$ are non-compact and complete in the metrics $g_{i}$. To conclude that $Q$ actually maps $\mathcal{C}^{\infty}\left(M_{2}\right)$ to $\mathcal{C}^{\infty}\left(M_{1}\right)$ we make use of the hypotheses on the injectivity radii and the Ricci tensor to apply standard Sobolev embedding theorems together with the following observations. 1 . The isometry of $Q$ as a map from $H^{1}\left(M_{2}, g_{2}\right)$ to $H^{1}\left(M_{1}, g_{1}\right)$ implies, by the fact that these spaces are the respective form domains of the operators $\Delta_{2}$ and $\Delta_{1}$, that $Q$ maps the domain of $\Delta_{2}$ to that of $\Delta_{1}$ and the intertwining relation (3.2) holds in the sense of operators. 2. The intertwining relation together with elliptic regularity theory for the Laplacians can be used to prove inductively that, if $u \in H_{0}^{2 k}\left(M_{2}, g_{2}\right)$, then $Q u \in H_{0}^{2 k}\left(M_{1}, g_{1}\right)$. The first observation already shows that this mapping property holds for $k=1$, and we can prove the general result by induction as follows. Suppose now that $Q$ maps $H_{0}^{2 k-2}\left(M_{2}, g_{2}\right)$ to $H_{0}^{2 k-2}\left(M_{1}, g_{1}\right)$. If $u \in H_{0}^{2 k}\left(M_{2}, g_{2}\right)$ for $k \geq 2$, we have $Q \circ \Delta_{2} u \in H_{0}^{2 k-2}\left(M_{1}, g_{1}\right)$ by hypothesis, so that $\Delta_{1}(Q u)=f \in H_{0}^{2 k-2}\left(M_{1}, g_{1}\right)$. It follows from elliptic regularity that $Q u \in H_{0}^{2 k}\left(M_{1}, g_{1}\right)$. Thus $Q$ maps $C^{\infty}\left(M_{2}\right) \cap L^{2}\left(M_{2}, g_{2}\right)$ to $\mathcal{C}^{\infty}\left(M_{1}\right) \cap L^{2}\left(M_{1}, g_{1}\right)$. 3. The map $Q$ is local in the sense that $Q: \mathcal{C}_{0}^{\infty}\left(M_{2}\right) \rightarrow \mathcal{C}_{0}^{\infty}\left(M_{1}\right)$ and if $\chi$ is a $T$ invariant cutoff function, $Q(\chi u)=\chi Q(u)$. Thus regularity is a local result and we can conclude that $Q$ extends to a map from $\mathcal{C}^{\infty}\left(M_{2}\right)$ to $\mathcal{C}^{\infty}\left(M_{1}\right)$.

Remark 3.6. In the application, $M_{1}=M_{2}=\mathbf{R}^{n}$, and the metrics $g_{1}$ and $g_{2}$ differ from the Euclidean metric on a compact set. In this case the assumptions in Proposition 3.3 on the injectivity radii and Ricci curvatures are trivially satisfied.

Theorem 3.7. Suppose a torus $T$ acts effectively on $\mathbf{R}^{n}$ by orthogonal transformations. Let $g_{1}$ and $g_{2}$ be compact perturbations of the Euclidean metric on $\mathbf{R}^{n}$ invariant under the action of $T$. Assume that the Riemannian measure defined by both metrics coincides with Lebesgue measure. Let $\left(\mathbf{R}^{n}\right)^{\prime}$ be the union of all principal orbits of the torus action. For each subtorus $K$ of $T$ of codimension at most one, suppose that there exists an orthogonal transformation $F_{K} \in O\left(\mathbf{R}^{n}\right)$ commuting with $T$ which induces an isometry $\overline{F_{K}}$ between the metrics induced by $g_{1}$ and $g_{2}$ on the quotient manifold $K \backslash\left(\mathbf{R}^{n}\right)^{\prime}$. Then $g_{1}$ and $g_{2}$ have the same scattering phase.

Remark 3.8. We have stated the theorem only in the form needed for the examples given here. However, the theorem may be generalized to other settings.

Proof. The manifolds $\left(\mathbf{R}^{n}, g_{1}\right)$ and $\left(\mathbf{R}^{n}, g_{2}\right)$ satisfy the hypotheses of Proposition 3.3. Define $Q: C^{\infty}\left(\mathbf{R}^{n}\right) \rightarrow C^{\infty}\left(\mathbf{R}^{n}\right)$ as in the conclusion of the proposition so that $\Delta_{1}=Q \circ \Delta_{2} \circ Q^{-1}$. Since the $F_{K}$ are orthogonal maps of $\mathbf{R}^{n}$, the map $Q$ induces an invertible isometry of $L^{2}\left(S^{n-1}\right)$. Since all orthogonal maps commute with the antipodal map of $S^{n-1}$, the map $Q_{\partial}$ commutes with $S_{0}(\lambda)$ as defined in equation 2.2.

¿From its construction, it is clear that the intertwining map $Q$ preserves the form of asymptotic expansions (2.1). Moreover, if $u$ is a solution $\left(\Delta_{g_{1}}-\lambda^{2}\right) u=0$ having an asymptotic expansion of the form (2.1), then $Q u$ is a solution of $\left(\Delta_{g_{2}}-\lambda^{2}\right) v=0$ having an asymptotic expansion of the form

$$
v(r \omega)=r^{(1-n) / 2} e^{i \lambda r} h_{+}(\omega)+r^{(1-n) / 2} e^{-i \lambda r} h_{-}(\omega)+\mathcal{O}\left(r^{-(n+1) / 2}\right)
$$

as $r \rightarrow \infty$, where

$$
h_{+}(\omega)=\left(Q_{\partial} f_{+}\right)(\omega)
$$


and

$$
h_{-}(\omega)=\left(Q_{\partial} f_{-}\right)(\omega) .
$$

Let $S_{g_{1}}(\lambda)$ and $S_{g_{2}}(\lambda)$ be the scattering matrices associated, respectively, to $\left(\mathbf{R}^{n}, g_{1}\right)$ and $\left(\mathbf{R}^{n}, g_{2}\right)$. Since

$$
f_{+}=S_{g_{1}}(\lambda) f_{-}
$$

and

$$
h_{+}=S_{g_{2}}(\lambda) h_{-}
$$

it follows from the uniqueness statement in Proposition 2.1 that

$$
Q_{\partial} S_{g_{1}}(\lambda) f_{-}=S_{g_{2}}(\lambda) Q_{\partial} f_{-} .
$$

Since this holds for any $f_{-} \in \mathcal{C}^{\infty}\left(S^{n-1}\right)$, and $Q_{\partial}$ is an invertible linear map, we have

$$
S_{g_{2}}(\lambda)=Q_{\partial} S_{g_{1}}(\lambda) Q_{\partial}^{-1} \text {. }
$$

Since $Q_{\partial}$ and $Q_{\partial}^{-1}$ commute with the operator $S_{0}(\lambda)$, we conclude that

$$
Q_{\partial} S_{g_{1}}(\lambda) S_{0}(\lambda)^{-1} Q_{\partial}^{-1}=S_{g_{2}}(\lambda) S_{0}(\lambda)^{-1}
$$

so that, on taking logarithms of determinants and using (2.4),

$$
\sigma_{g_{2}}(\lambda)=\sigma_{g_{1}}(\lambda) .
$$

\section{Examples}

In [9], the first author constructed continuous families of Riemannian metrics on $\mathbf{R}^{n}, n \geq 9$, which pairwise satisfy the hypotheses of Theorem 3.7 modulo the condition that the metrics be Euclidean outside of a compact set. Dorothee Schueth [24] pointed out that the metrics could be modified to satisfy this additional condition; in fact they could be flat outside of a compact set of arbitrarily small volume. Moreover, Schueth constructed new continuous families of metrics on $\mathbf{R}^{n}, n \geq 8$, pairwise satisfying the condition of Theorem 3.7. (Note that she lowered the minimum dimension by one.) Additionally, Schueth constructed pairs, though not continuous families, of such metrics on $\mathbf{R}^{6}$. In both these papers, the focus was on compact manifolds. The metrics, once constructed, were restricted to the unit ball and sphere. Using Proposition 3.3, these restricted metrics were seen to be isospectral. In the present context, we will conclude from Theorem 3.7 that the families of metrics on $\mathbf{R}^{n}$ constructed in these two papers are isosphasal.

We now review the construction of the metrics in [9] modified as in [24].

Definition 4.1. (i) We will say that two skew-symmetric bilinear maps $[\cdot, \cdot]$ and $[\cdot, \cdot]^{\prime}$ taking $\mathbf{R}^{m} \times \mathbf{R}^{m}$ to $\mathbf{R}^{k}$ are isospectral if for each $Z \in \mathbf{R}^{k}$ there is an orthogonal transformation $A_{Z}$ with the property that for every pair of vectors $(x, y) \in \mathbf{R}^{m} \times \mathbf{R}^{m}$,

$$
\left\langle[x, y]^{\prime}, Z\right\rangle_{\mathbf{R}^{k}}=\left\langle\left[A_{Z} x, A_{Z} y\right], Z\right\rangle_{\mathbf{R}^{k}},
$$

where, here and in what follows, $\langle\cdot, \cdot\rangle_{\mathbf{R}^{k}}$ denotes the Euclidean inner product on $\mathbf{R}^{k}$. (ii) We will say that the skew-symmetric bilinear maps $[\cdot, \cdot]$ and $[\cdot, \cdot]^{\prime}$ are equivalent if there exists an orthogonal transformation $A$ of $\mathbf{R}^{m}$ and an orthogonal transformation $C$ of $\mathbf{R}^{k}$, which preserves the lattice $(2 \pi \mathbf{Z})^{k}$, such that $\left\langle[A x, A y]^{\prime}, Z\right\rangle=$ 
$\langle[x, y], C Z\rangle$ for all $x, y \in \mathbf{R}^{m}$ and $Z \in \mathbf{R}^{k}$. We will also say that the pair of maps $(A, C)$ is an equivalence of $[\cdot, \cdot]$ and $[\cdot, \cdot]^{\prime}$ in this case.

Remark 4.2. (i) Our notation differs from that of [9]. The bilinear maps $[\cdot, \cdot]$ : $\mathbf{R}^{m} \times \mathbf{R}^{m} \rightarrow \mathbf{R}^{k}$ correspond to linear maps $j: \mathbf{R}^{k} \rightarrow \mathfrak{s o}(m)$ via

$$
\langle[x, y], Z\rangle=\langle j(Z) x, y\rangle
$$

for all $x, y \in \mathbf{R}^{m}$ and $Z \in \mathbf{R}^{k}$. We say that $j$ and $j^{\prime}$ are isospectral if $j^{\prime}(Z)$ and $j(Z)$ are isospectral linear operators for each $z \in \mathbf{R}^{k}$. We will say that $j$ and $j^{\prime}$ are equivalent if there exist orthogonal maps $A$ of $\mathbf{R}^{m}$ and $C$ of $\mathbf{R}^{m}$ such that $C$ preserves the lattice $(2 \pi \mathbf{Z})^{k}$ and such that $A j^{\prime}(Z) A^{-1}=j(C Z)$ for all $z \in \mathbf{R}^{k}$. These conditions correspond to the isospectrality and equivalence conditions in Definition 4.1. The $j$ maps were used in [9] rather than the bracket maps [, ].

(ii) Our notion of equivalence differs slightly from that in [9] and [10] in that we require $C$ to preserve the lattice $(2 \pi \mathbf{Z})^{k}$. This condition is added so that $C$ induces a transformation of the torus $(2 \pi \mathbf{Z})^{k} \backslash \mathbf{R}^{k}$.

Definition 4.3. (i) Let $T$ be the $k$-torus $(2 \pi \mathbf{Z})^{k} \backslash \mathbf{R}^{k}$ embedded in $S O(2 k)$ as $S O(2) \times$ $\cdots \times S O(2)$. Then $T$ acts on $\mathbf{R}^{2 k}=\mathbf{R}^{2} \times \cdots \times \mathbf{R}^{2}$ by the standard $S O(2)$-action in each factor. This action is not free but is inner-product preserving. The Lie algebra of $T$ is $\mathfrak{z}=\mathfrak{s o}(2) \oplus \cdots \oplus \mathfrak{s o}(2) \simeq \mathbf{R}^{k}$. Given $Z \in \mathfrak{z}$, define a vector field $Z^{*}$ on $\mathbf{R}^{2 k}$ by

$$
Z_{u}^{*}=\left.\frac{d}{d t}\right|_{t=0}(\exp (t Z) \cdot u)
$$

for $u \in \mathbf{R}^{2 k}$. Observe that, for $x, y \in \mathbf{R}^{m},[x, y] \in \mathbf{R}^{k}=\mathfrak{z}$ so that we may use (4.1) to define a vector field $[x, y]^{*}$ on $\mathbf{R}^{2 k}$.

(ii) Given a bilinear map $[\cdot, \cdot]: \mathbf{R}^{m} \times \mathbf{R}^{m} \rightarrow \mathbf{R}^{k}$ and a smooth, compactly supported function $\varphi:[0, \infty) \times[0, \infty) \rightarrow[0, \infty)$, we now construct a Riemannian metric $g=$ $g^{[\cdot, \cdot], \varphi}$ on $\mathbf{R}^{m+2 k}$. Denote elements of $\mathbf{R}^{m+2 k}$ by $(x, u)$ with $x \in \mathbf{R}^{m}$ and $u \in \mathbf{R}^{2 k}$. First define $\psi: \mathbf{R}^{m+2 k} \rightarrow[0, \infty)$ by $\psi(x, u)=\varphi\left(\|x\|^{2},\|u\|^{2}\right)$. For $(x, u) \in \mathbf{R}^{m+2 k}$, denote by $(Y, W)$ a typical element of the tangent space $T_{(x, u)} \mathbf{R}^{m+2 k}$, where, by standard identifications, $Y \in \mathbf{R}^{m}$ and $W \in \mathbf{R}^{2 k}$. We set

$$
g((0, W),(0, V))=\langle W, V\rangle_{\mathbf{R}^{2 k}}
$$

and define the $g$-orthogonal complement to $\{0\} \oplus \mathbf{R}^{2 k}$ in $T_{(x, u)} \mathbf{R}^{m+2 k}$ as follows. For $(x, u) \in \mathbf{R}^{m} \times \mathbf{R}^{2 k}$ and $Y \in T_{x} \mathbf{R}^{m}$, let

$$
\tilde{Y}_{x, u}=(Y, Z)
$$

with

$$
Z=\psi(x, u)[x, Y]_{u}^{*} .
$$

The $g$-orthogonal complement to $\{0\} \oplus \mathbf{R}^{2 k}$ is taken to be

$$
\left\{\tilde{Y}_{x, u}: Y \in T_{x} \mathbf{R}^{m}\right\} \text {. }
$$

We put an inner product on this space so that the map $Y \mapsto \tilde{Y}_{x, u}$ is an isometry where $\mathbf{R}^{m}$ has the Euclidean inner product. 
Note that, for $(x, u)$ outside of the support of $\psi$, we have $\tilde{Y}_{x, u}=Y$. Thus the metric so constructed is identical to the Euclidean metric away from the support of $\psi$.

Proposition 4.4. Suppose that $[\cdot, \cdot]$ and $[\cdot, \cdot]^{\prime}$ are isospectral in the sense of Definition 4.1 and that $g$ and $g^{\prime}$ are metrics constructed as in Definition 4.3 from the data $(\varphi,[\cdot, \cdot])$ and $\left(\varphi,[\cdot, \cdot]^{\prime}\right)$ for the same nonnegative function $\varphi \in \mathcal{C}_{0}^{\infty}\left(\mathbf{R}^{+} \times \mathbf{R}^{+}\right)$. Then $g$ and $g^{\prime}$ are isophasal.

Proof. We apply Theorem 3.7. Let $W$ denote the union of the principal orbits for the action of $T$ on $\mathbf{R}^{2 k}$. By identifying $\mathbf{R}^{2 k}$ with $\mathbf{C}^{k}$, we may write

$$
W=\left\{\left(z_{1}, \ldots, z_{k}\right) \in \mathbf{C}^{k}: z_{1} \neq 0, \ldots, z_{k} \neq 0\right\} .
$$

The union of the principal orbits for the action of $T$ on $\mathbf{R}^{m+2 k}$ is given by $\mathbf{R}^{m} \times W$.

If $K \subset T$ is a subtorus of codimension one, then in the Lie algebra $\mathfrak{z}$ of $T$, there is a vector $Z$ orthogonal to the Lie subalgebra $\mathfrak{k}$ of $K$. By hypothesis, there is an orthogonal transformation $A_{Z} \in O(m)$ so that

$$
\left\langle[x, y]^{\prime}, Z\right\rangle_{\mathbf{R}^{k}}=\left\langle\left[A_{Z} x, A_{Z} y\right], Z\right\rangle_{\mathbf{R}^{k}}
$$

for any $x$ and $y$ belonging to $\mathbf{R}^{m}$. Letting $g_{K}$ and $g_{K}^{\prime}$ be the metrics on $K \backslash\left(\left(\mathbf{R}^{m}\right)^{\prime} \times W\right)$ induced by $g$ and $g^{\prime}$, it follows from Definition 4.3(ii) that the orthogonal map

$$
\tau_{K}(x, u)=\left(A_{Z} x, u\right)
$$

of $\mathbf{R}^{m+2 k}$ induces an isometry from $\left(K \backslash\left(\left(\mathbf{R}^{m}\right)^{\prime} \times W\right), g_{K}\right)$ to $\left(K \backslash\left(\left(\mathbf{R}^{m}\right)^{\prime} \times W\right), g_{K}^{\prime}\right)$. Thus the hypotheses of Theorem 3.7 are satisfied, and we conclude that the metrics are isophasal.

Remark 4.5. In [9], the function $\psi$ did not appear; i.e., $\varphi$ (and thus $\psi$ ) was identically one. As mentioned above, it was Dorothee Schueth that realized the function $\psi$ could be inserted so that the metrics are Euclidean outside of a compact set.

By referring to the proofs of Proposition 4.4 and of Theorem 3.7, we can give an explicit description of the intertwining operators between the Laplacians of the metrics in Proposition 4.4 and between their scattering phases as follows:

Proposition 4.6. Define $g$ and $g^{\prime}$ as in Proposition 4.4. Writing $\mathbf{R}^{2 k}=\mathbf{R}^{2} \times \cdots \times \mathbf{R}^{2}$ and letting $\left(r_{i}, \theta_{i}\right)$ denote polar coordinates on the ith factor, we obtain coordinates $(x, r, \theta)$ on $\mathbf{R}^{m+2 k}$, where $x=\left(x_{1}, \ldots, x_{m}\right), r=\left(r_{1}, \ldots, r_{k}\right)$ and $\theta=\left(\theta_{1}, \ldots, \theta_{k}\right)$. For $Z \in \mathbf{R}^{k}$, choose $A_{Z}$ as in Definition 4.1. Define $Q: C^{\infty}\left(\mathbf{R}^{m+2 k}\right) \rightarrow C^{\infty}\left(\mathbf{R}^{m+2 k}\right)$ by

$$
Q(f)(x, r, \theta)=\sum_{Z \in\left(\frac{1}{2 \pi} \mathbf{Z}\right)^{k}}(2 \pi)^{-k / 2}\left(\int_{[0,2 \pi]^{k}} f\left(A_{Z}(x), r, \sigma\right) e^{-i Z \cdot \sigma} d \sigma\right) e^{i Z \cdot \theta} .
$$

Then $Q$ intertwines the Laplacians of the metrics $g$ and $g^{\prime}$ on $\mathbf{R}^{m+2 k}$, and the associated map $Q_{\partial}$, defined as in Theorem 3.7, intertwines the associated scattering operators.

We now consider whether these metrics are isometric.

Proposition 4.7. Fix $\varphi$. Let $g$ and $g^{\prime}$ be the metrics defined as in Definition 4.3 by nontrivial maps $[\cdot, \cdot]$ and $[\cdot, \cdot]^{\prime}: \mathbf{R}^{m} \times \mathbf{R}^{m} \rightarrow \mathbf{R}^{k}$ together with $\varphi$. 
(i) Suppose that $\tau$ is an isometry from $\left(\mathbf{R}^{m+2 k}, g\right)$ to $\left(\mathbf{R}^{m+2 k}, g^{\prime}\right)$ that carries T-orbits to T-orbits. Then $\tau$ is of the form

$$
(\tau(x, u)=(A(x), \widetilde{C}(u))
$$

where $A \in O(m), \widetilde{C} \in O(2 k)$, and $\widetilde{C}$ normalizes $T$. Letting $C$ be the automorphism of the Lie algebra $\mathbf{R}^{k}=\mathfrak{s o}(2) \oplus \cdots \oplus \mathfrak{s o}(2)$ of $T$ given by conjugation by $\widetilde{C}$, then the pair $(A, C)$ is an equivalence of $[\cdot, \cdot]$ and $[\cdot, \cdot]^{\prime}$, as in Definition 4.1.

(ii) Conversely, every map $\tau$ of this form is an isometry between the two metrics.

Proof. (ii) is straightforward and is left to the reader.

(i) Since the isometry $\tau$ carries $T$-orbits to $T$-orbits, it must preserve the open dense subset $\mathbf{R}^{m} \times W$, where $W$ is given in the proof of Proposition 4.4. The submanifold $\mathbf{R}^{m} \times W$ has the structure of a principal $T$ bundle over $\mathbf{R}^{m} \times(T \backslash W) \simeq \mathbf{R}^{m} \times\left(\mathbf{R}^{+}\right)^{k}$. The metrics $g$ and $g^{\prime}$ both induce the standard Euclidean metric on the quotient $\mathbf{R}^{m} \times\left(\mathbf{R}^{+}\right)^{k}$. The isometry $\tau$ induces an isometry $\bar{\tau}$ of $\mathbf{R}^{m} \times\left(\mathbf{R}^{+}\right)^{k}$. Such an isometry is the composition of a translation in $\mathbf{R}^{m}$ with an orthogonal transformation of the form $A \times P$, where $A \in O(m)$ and $P$ permutes the coordinates in $\left(\mathbf{R}^{+}\right)^{k}$. We claim that the translation factor is trivial. To see this, note that the metrics $g$ and $g^{\prime}$ on $\mathbf{R}^{m+2 k}$ are Euclidean on the complement of $\left\{(x, u) \in \mathbf{R}^{m+2 k}:(\|x\|,\|u\|) \in \operatorname{supp}(\varphi)\right\}$. Letting $R$ be minimal such that $\operatorname{supp}(\varphi) \subset\left\{(s, t): s^{2}+t^{2} \leq R\right\}$, then $g$ and $g^{\prime}$ are Euclidean on the region $\left\{(x, u):\|x\|^{2}+\|u\|^{2} \geq R\right\}$ and not on any translate of this region. Hence $\bar{\tau}$ must preserve the image of this region in $\mathbf{R}^{m} \times\left(\mathbf{R}^{+}\right)^{k}$, and the claim follows.

For each $x \in \mathbf{R}^{m}, \tau$ restricts to an isometry from the Euclidean space $\{x\} \times \mathbf{R}^{2 k}$ to the Euclidean space $\{A(x)\} \times \mathbf{R}^{2 k}$. Canonically identifying both spaces with $\mathbf{R}^{2 k}$, this isometry preserves the origin, since the origin is the unique $T$-orbit which is a single point. Thus $\tau$ is of the form $\tau(x, u)=\left(A(x), B_{x}(u)\right)$ with $B_{x} \in \mathrm{O}(2 k)$ for each $x \in \mathbf{R}^{m}$. We may identify $T$ with the maximal torus $T=\mathrm{SO}(2) \times \cdots \times \mathrm{SO}(2)$ of $\mathrm{O}(2 k)$. Since $\tau$ carries $T$-orbits to $T$-orbits, each $B_{x}$ must normalize $T$. Noting that $T$ has finite index in its normalizer in $\mathrm{O}(2 k)$ and that $B_{x}$ depends smoothly on $x$, there must exist $\widetilde{C} \in \mathrm{O}(2 k)$, independent of $x$, and $z(x) \in T$ such that $B_{x}=z(x) \circ \widetilde{C}$. The permutation $P$ in the expression for $\bar{\tau}$ is the map of $T \backslash W$ induced by $\widetilde{C}$.

We next show that $(A, C)$ defines an equivalence of $[\cdot, \cdot]$ and $[\cdot, \cdot]^{\prime}$, where $C$ is defined from $\widetilde{C}$ as in the statement of the proposition. Observe that $\tau \circ \psi=\tau$, since $\tau(x, u)$ preserves the norms of the two coordinates. Since $\tau_{*}$ maps $g$-horizontal vectors at each point $(x, u)$ (i.e., vectors $g$-orthogonal to the orbit of $T$ through $(x, u))$ to $g^{\prime}$ horizontal vectors through $\tau(x, u)$, we have for $y \in \mathbf{R}^{m}$, viewed as a tangent vector to $\mathbf{R}^{m}$ at $x$,

$$
\tau_{*(x, u)}\left(y+\psi(x, u)[x, y]_{(x, u)}^{*}\right)=A(y)+\psi(x, u)[A(x), A(y)]_{(\tau(x, u)}^{*} .
$$

Noting that $\tau_{*}\left(Z^{*}\right)=C\left(Z^{*}\right)$ for $Z$ in the Lie algebra $\mathfrak{z}=\mathbf{R}^{k}$ of $T$, it follows that $[A(x), A(y)]^{*}=\left(C([x, y])^{*}\right.$. Since the map $Z \rightarrow Z^{*}$ is injective on $\mathfrak{z}$, we see that $(A, C)$ is an equivalence of $[\cdot, \cdot]$ and $[\cdot, \cdot]^{\prime}$.

It remains to show that the map $x \rightarrow z(x)$ is constant. Fix a point $x_{0}$ and let $z=z\left(x_{0}\right)$. Define $\mu(x, u)=(A(x), z \cdot \widetilde{C}(u))$. By (ii), $\mu$ is an isometry from $g$ to $g^{\prime}$. Hence $\alpha:=\tau^{-1} \circ \mu$ is an isometry of $g$ of the form $(x, u) \rightarrow(x, w(x) \cdot u)$ for some 
map $w: \mathbf{R}^{m} \rightarrow T$ satisfying $w\left(x_{0}\right)=1$, where 1 denotes the identity element in $T$. At points of the form $\left.\left(x_{0}, u\right)\right), u \in \mathbf{R}^{2 k}$, the differential $\alpha_{*}$ acts as the identity both on the tangent space to the fiber and on the horizontal space. Thus $\alpha_{*\left(x_{0}, u\right)}=I d$. Since an isometry is uniquely determined by its value and its differential at a single point, it follows that $\alpha=I d$, i.e., that $z(x) \equiv z$. Replacing $\widetilde{C}$ by $z \cdot \widetilde{C}$, the proposition follows.

Corollary 4.8. Let $g$ be the Riemannian metric on $\mathbf{R}^{m+2 k}$ defined from the data $([\cdot, \cdot], \varphi)$ as in Definition 4.3. Then the centralizer of $T$ in the group of all isometries of $g$ consists of all maps $\tau$ of $\mathbf{R}^{m+2 k}$ of the form $\tau(x, u)=(A(x), z \cdot u)$ such that $A \in \mathrm{O}(m)$ preserves $[\cdot, \cdot]$ (i.e., the pair $(A, I d)$ is a self-equivalence of $[\cdot, \cdot]$ ) and such that $z \in T$.

Proof. An isometry that commutes with $T$ must carry $T$-orbits to $T$-orbits. Thus the corollary follows from Proposition 4.7 and the fact that $T$ is its own centralizer in $\mathrm{O}(2 k)$.

Proposition 4.9. Suppose that $g$ and $g^{\prime}$ are metrics constructed from the data $([\cdot, \cdot], \varphi)$ and $\left([\cdot, \cdot]^{\prime}, \varphi\right)$ as in Definition 4.3 , where $[\cdot, \cdot]$ and $[\cdot, \cdot]^{\prime}$ are inequivalent in the sense of Definition 4.1. Assume that $[\cdot, \cdot]$ satisfies the genericity condition that $[\cdot, \cdot \cdot]$ is invariant under only finitely many orthogonal transformations of $\mathbf{R}^{m}$. Then $g$ is not isometric to $g^{\prime}$.

Proof. By Corollary 4.8 and the genericity condition on $[\cdot, \cdot], T$ is a maximal torus in the full isometry group of $g$. Now suppose that $\rho:\left(\mathbf{R}^{n}, g\right) \rightarrow\left(\mathbf{R}^{n}, g^{\prime}\right)$ is an isometry. Since the metrics are isometric, $T$ must also be a maximal torus in the full isometry group of $g^{\prime}$. By the conjugacy of the maximal tori in any Lie group, we may assume after composing with an isometry of $g^{\prime}$ that $\rho$ carries $T$-orbits to $T$-orbits. By Proposition 4.7 , it follows that $[\cdot, \cdot]$ and $[\cdot, \cdot]^{\prime}$ are equivalent, contradicting the hypothesis.

By Proposition 4.4 and Proposition 4.9 , if $[\cdot, \cdot]$ and $[\cdot, \cdot]^{\prime}$ are isospectral, inequivalent maps $\mathbf{R}^{m} \times \mathbf{R}^{m} \rightarrow \mathbf{R}^{k}$ in the sense of Definition 4.1 and if $[\cdot, \cdot \cdot]$ satisfies the genericity condition of Proposition 4.9, then the metrics on $\mathbf{R}^{m+2 k}$ constructed from the data $([\cdot, \cdot], \varphi)$ and $\left([\cdot, \cdot]^{\prime}, \varphi\right)$ as in Definition 4.3 , for any fixed choice of $\varphi$, are isospectral but not isometric. The following lemma shows that such isospectral, inequivalent maps are plentiful.

Proposition 4.10. [10] Let $k=2$, and let $m$ be any positive integer other than $1,2,3,4$, or 6 . Let $W_{m}$ be the real vector space consisting of all anti-symmetric bilinear maps from $\mathbf{R}^{m} \times \mathbf{R}^{m}$ to $\mathbf{R}^{k}$. Then there is a Zariski open subset $\mathcal{O}_{m}$ of $W_{m}$ (i.e., $\mathcal{O}_{m}$ is the complement of the zero locus of some non-zero polynomial function on $W$ ) such that each $[\cdot, \cdot] \in \mathcal{O}_{m}$ belongs to a d-parameter family of isospectral, inequivalent elements of $W_{m}$. Here $d \geq \frac{m(m-1)}{2}-\left[\frac{m}{2}\right]\left(\left[\frac{m}{2}\right]+2\right)>1$. In particular, $d$ is of order at least $\mathcal{O}\left(\mathrm{m}^{2}\right)$. Moreover, the elements of $\mathcal{O}_{m}$ satisfy the genericity condition of Proposition 4.9.

The statement of the proposition in [10] is in the language of Remark 4.2. The final statement of the proposition was not explicitly stated in [10]; however, a glance at the proof given there shows that the genericity condition is one of the defining 
properties of the Zariski open set $\mathcal{O}_{m}$ constructed there. While the proposition omits $m=6$, an explicit example of a continuous family of isospectral, inequivalent maps $\mathbf{R}^{m} \times \mathbf{R}^{m} \rightarrow \mathbf{R}^{2}$ was also constructed in [10].

We have now proven Theorem 1.1 when $n \geq 9$. (For $n=10$, we refer to the comment immediately above.)

To prove Theorem 1.1 in the case $n=8$ for continuous families and $n=6$ for pairs, we refer to the article [24] by Dorothee Schueth. There Schueth constructed metrics on $\mathbf{R}^{n}$ from data $(L, \psi)$ consisting of a particular type of linear map $L$ and a cut-off function $\psi$ on $\mathbf{R}^{n}$ of the same type used above. (The maps $L$, which play the role of the $[\cdot, \cdot]$ maps in the construction above, are denoted $j$ or $c$ in the two different constructions given in [24].) She defined notions of isospectrality and equivalence of the linear maps. An argument analogous to the proof of Proposition 4.4 shows that, for fixed $\psi$, the metrics on $\mathbf{R}^{n}$ constructed from isospectral linear maps $L$ and $L^{\prime}$ are isosphasal. To discuss the condition for non-isometry, we will for simplicity require that the cut-off function $\psi$ be radial. The metrics constructed in [24] are Euclidean outside of the support of $\psi$ but not on any open set on which $\psi$ is positive. Since $\psi$ is supported on a ball $B$ about the origin, any isometry between the metrics must therefore carry this ball to itself. Under a genericity condition analogous to that in Proposition 4.9, Schueth proved that the metrics on the ball are not isometric provided that the associated linear maps are inequivalent. This completes the proof.

Example 4.11. We give an explicit triple of isophasal metrics on $\mathbf{R}^{12}$ and compare their geometries. We let $k=3$ and $m=6$. Define three maps $[\cdot, \cdot]_{i}: \mathbf{R}^{6} \times \mathbf{R}^{6} \rightarrow \mathbf{R}^{3}$ as follows.

To define $[\cdot, \cdot]_{1}$ and $[\cdot, \cdot]_{2}$, view $\mathbf{R}^{6}$ as $\mathbf{R}^{3} \times \mathbf{R}^{3}$ and denote elements of $\mathbf{R}^{6}$ as ordered pairs $(x, y)$, with $x, y \in \mathbf{R}^{3}$. Let $\times$ denote the cross product on $\mathbf{R}^{3}$. Define

$$
\left[(x, y),\left(x^{\prime}, y^{\prime}\right)\right]_{1}=x \times x^{\prime}+y \times y^{\prime}
$$

and

$$
\left[(x, y),\left(x^{\prime}, y^{\prime}\right)\right]_{2}=x \times x^{\prime}-y \times y^{\prime} .
$$

To define $[\cdot, \cdot]_{3}$, view $\mathbf{R}^{6}$ as $H \times \mathbf{R}^{2}$, where $H$ denotes the quaternions. Denote elements of $\mathbf{R}^{6}$ as pairs $(q, y)$, with $q \in H, y \in \mathbf{R}^{2}$. View the target space $\mathbf{R}^{3}$ as the purely imaginary quaternions. Define

$$
\left[(q, y),\left(q^{\prime}, y^{\prime}\right)\right]_{3}=\operatorname{Im}\left(q q^{\prime}\right)
$$

where $q q^{\prime}$ is the quaternionic product.

To see that the three bracket maps are isospectral, it is easier to consider the associated maps $j_{i}: \mathbf{R}^{3} \rightarrow \mathfrak{s o}(6)$ defined as in Remark 4.2. We have

$$
\begin{gathered}
j_{1}(z)(x, y)=(z \times x, z \times y), \\
j_{2}(z)(x, y)=(z \times x,-z \times y),
\end{gathered}
$$

and

$$
j_{3}(z)(q, y)=(z q, 0),
$$

where in the final equation, $z q$ denotes quaternionic multiplication of the purely imaginary quaternion $z$ with the quaternion $q$. In each case, the eigenvalues of $j_{i}(z)$ are $\|z\| \sqrt{-1},-\|z\| \sqrt{-1}$, and 0 , each occurring with multiplicity 2 . Thus $j_{1}(z), j_{2}(z)$ and $j_{3}(z)$ are similar transformations for each $z$, and hence the $j_{i}$ are mutually isospectral. 
Equivalently, the $[\cdot, \cdot]_{i}$ are mutually isospectral. Thus fixing a choice of $\varphi$, we obtain a triple of isosphasal metrics $g_{i}$ on $\mathbf{R}^{12}$.

The isometry groups Iso $\left(g_{i}\right)$ of the three metrics vary both in their dimension and structure. By Corollary 4.8 and Remark 4.2, every isometry of $g_{i}$ that commutes with $T$ is the composition of an element of $T$ with an isometry of the form $A \times \operatorname{Id}$ acting on $\mathbf{R}^{12}=\mathbf{R}^{6} \times \mathbf{R}^{6}$, where $A \in \mathrm{O}(6)$ commutes with all the $j_{i}(z), z \in \mathbf{R}^{k}$. The image of $j_{1}$ in $\mathfrak{s o}(6)$ is the set of all matrices of the form

$$
\left(\begin{array}{cc}
B & 0 \\
0 & B
\end{array}\right)
$$

with $B \in \mathfrak{s o}(3)$. The connected component of the centralizer of this image in $\mathrm{O}(6)$ is the one-parameter subgroup (circle) generated by the skew-symmetric matrix

$$
\left(\begin{array}{cc}
0 & \mathrm{Id} \\
-\mathrm{Id} & 0
\end{array}\right)
$$

Thus the identity component of the centralizer of $T$ in $\operatorname{Iso}\left(g_{1}\right)$ is isomorphic to $T \times$ $S^{1}$, a four-dimensional torus. In particular, the maximal tori in $\operatorname{Iso}\left(g_{1}\right)$ are fourdimensional.

The image of $j_{2}$ in $\mathfrak{s o}(6)$ is the set of all matrices of the form

$$
\left(\begin{array}{cc}
B & 0 \\
0 & -B
\end{array}\right)
$$

with $B \in \mathfrak{s o}(3)$. The connected component of the centralizer of this image in $\mathrm{O}(6)$ is trivial. Thus the three-dimensional torus $T$ is a maximal torus in $\operatorname{Iso}\left(g_{2}\right)$ and is the identity component of its own centralizer.

The connected component of the centralizer of the image of $j_{3}$ in $\mathfrak{s o}(6)$ is isomorphic to $S U(2) \times S O(2)$, where the 3 -sphere $S U(2)$ is identified with the unit quaternions acting on $H$ by right multiplication and where $S O(2)$ acts on the $\mathbf{R}^{2}$ factor. Since a maximal torus in $S U(2) \times S O(2)$ is two-dimensional, the maximal tori in $\operatorname{Iso}\left(g_{3}\right)$ are five-dimensional. Moreover, the semisimple group $S U(2) \times S O(2)$ acts by isometries preserving the bundle structure.

\section{Existence of resonances}

We will show that, generically, the metrics we have constructed on $\mathbf{R}^{n}$ have infinitely many scattering resonances, in contrast to the Euclidean Laplacian on $\mathbf{R}^{n}$ which has none. By results of Sá Barreto-Tang [21] for $n$ odd and of Tang [28] for $n$ is even, it suffices to verify that the second heat invariant $a_{2}$ is nonzero. Indeed, for $n$ even, Tang showed that, if a metric has only finitely many resonances, then all the heat invariants $a_{k}$ for $k \geq 2$ must vanish. For $n$ odd, Barreto-Tang showed that if the Laplacians of two metrics on $\mathbf{R}^{n}$, which differ from the Euclidean metric by a superexponentially decaying perturbation, have the same resonances, then they also have the same heat invariants $a_{k}$ for $k \geq 2$. This implies that a metric with non-vanishing heat invariant $a_{2}$ must have at least finitely many resonances, and a closer analysis of the renormalized wave trace shows that, in fact, the number of resonances must be infinite in this case.

Here, we carry out the proof that the heat invariant $a_{2}$ is non-zero for the specific metrics constructed in Section 4. For convenience, we will restrict to the cases that 
$k=2$ or $k=3$ in the notation of Section 4 . These two cases include all the examples constructed by Proposition 4.10 as well as Example 4.11.

Recall that for any Riemannian metric $g$ on an $n$-dimensional manifold $M$, the second heat invariant is given by

$$
a_{2}(g)=\frac{(4 \pi)^{-\frac{n}{2}}}{360} \int_{M}\left(5 \tau^{2}-2\|\operatorname{Ric}\|^{2}+2\|R\|^{2}\right) \operatorname{dvol}_{g},
$$

where $\tau$ denotes the scalar curvature.

Notation 5.1. Fix a non-trivial bilinear map [., $\cdot]: \mathbf{R}^{m} \times \mathbf{R}^{m} \rightarrow \mathbf{R}^{k}$. Given $\varphi$ : $[0, \infty) \times[0, \infty) \rightarrow[0, \infty)$, consider the family of functions $\varphi_{s}, s \in \mathbf{R}^{+}$, given by $\varphi_{s}\left(t_{1}, t_{2}\right)=\varphi\left(t_{1}, s^{2} t_{2}\right)$. Set

$$
g^{s}=g^{[\cdot, \cdot], \varphi_{s}}
$$

as in Definition 4.3 and denote by $R^{(s)}$ and $\operatorname{Ric}^{(s)}$ the curvature tensor and Ricci tensor of the metric $g^{s}$.

Theorem 5.2. Let $k=2$ or 3 , let $[\cdot, \cdot]: \mathbf{R}^{m} \times \mathbf{R}^{m} \rightarrow \mathbf{R}^{k}$ be a non-zero bilinear map, and let $\varphi:[0, \infty) \times[0, \infty) \rightarrow[0, \infty)$ be a non-trivial compactly supported $\mathcal{C}^{\infty}$ function. We use Notation 5.1. Except for possibly finitely many values of $s, a_{2}\left(g^{s}\right) \neq 0$.

Definition 5.3. Let $\left\{f^{s}\right\}, s \in \mathbf{R}^{+}$, be a one-parameter family of functions on $\mathbf{R}^{m+2 k}$. We will say that $\left\{f^{s}\right\}$ is a homogeneous $(r, s)$-deformation of degree $d$ if $f^{s}(x, r, \theta)=$ $s^{d} f^{1}(x, \tilde{r}, \theta)$ for all $s$, where $\tilde{r}=\left(\tilde{r}_{1}, \ldots, \tilde{r}_{k}\right)=\left(s r_{1}, \ldots, s r_{k}\right)$. We will say $\left\{f^{s}\right\}$ is an $(r, s)$-deformation of degree $d$ if $f^{s}=f_{1}^{s}+\ldots f_{l}^{s}$ where each $\left\{f_{i}^{s}\right\}$ is a homogeneous $(r, s)$-deformation, say of degree $d_{i}$, and where $d=\max \left\{d_{i}\right\}$. Assuming the $d_{i}$ 's are distinct, we will refer to $f_{i}^{s}$ as the homogeneous term of degree $d_{i}$ in $f^{s}$.

The following two lemmas are elementary.

Lemma 5.4. If $\left\{f^{s}\right\}$ is a homogeneous $(r, s)$-deformation of degree $d$, then:

(i) $\left\{\frac{\partial}{\partial x_{j}} f^{s}\right\}, 1 \leq j \leq m$, is also a homogeneous $(r, s)$-deformation of degree $d$ while $\left\{\frac{\partial}{\partial r_{p}} f^{s}\right\}, 1 \leq p \leq k$, is a homogeneous $(r, s)$-deformation of degree $d+1$;

(ii) $\left\{r_{p} f^{s}\right\}, 1 \leq p \leq k$, is a homogeneous $(r, s)$-deformation of degree $d-1$.

(iii) If $\left\{h^{s}\right\}$ is a homogeneous $(r, s)$-derivation of degree $d^{\prime}$, then $\left\{f^{s} h^{s}\right\}$ is a homogeneous $(r, s)$-deformation of degree $d+d^{\prime}$.

Lemma 5.5. If $\left\{f^{s}\right\}$ is a homogeneous $(r, s)$-deformation of degree $d$ and if each $f^{s}$ is continuous with compact support, then

$$
\int_{\mathbf{R}^{m+2 k}} f^{s}=s^{d-2 k} \int_{\mathbf{R}^{m+2 k}} f^{1}
$$

where the integrals are with respect to Lebesgue measure.

To prove that the function $a_{2}\left(g^{s}\right)$ is non-zero except for possibly finitely many choices of $s$, we will show that the integrand is an $(r, s)$-deformation of degree 2 , and that the homogeneous term of degree two is strictly positive. Consequently, after multiplying by an appropriate power of $s$, the heat invariant $a_{2}\left(g^{s}\right)$ is a non-trivial polynomial in $s$, from which Theorem 5.2 follows. 
Notation 5.6. (i) Write $\mathbf{R}^{2 k}$ as $\mathbf{R}^{2} \times \mathbf{R}^{2} \cdots \times \mathbf{R}^{2}$ and let $\left(r_{i}, \theta_{i}\right), i=1,2, \ldots, k$, denote the polar coordinates on the $k$ factors $\mathbf{R}^{2}$. We thus coordinatize a dense open subset of $\mathbf{R}^{m+2 k}$ by $(x, r, \theta)=\left(x_{1}, \ldots, x_{m}, r_{1}, r_{2}, \ldots r_{k}, \theta_{1}, \theta_{2}, \ldots, \theta_{k}\right)$.

(ii) For $\left\{Z_{1}, \ldots, Z_{k}\right\}$ the standard basis of $\mathfrak{z}=\mathbf{R}^{k}$, the vector field $Z_{p}^{*}, p=1, \ldots, k$, defined in equation (4.1), is given by $\frac{\partial}{\partial \theta_{p}}$.

(iii) Let indices $i, j, k$ run from 1 to $m$, indices $p, q$ range over $1,2, \ldots, k$, and Greek indices range over $1, \ldots, m+2 k$. Define an orthonormal frame field as follows: For $p=1,2, \ldots, k$, let $\hat{r}_{p}=\frac{\partial}{\partial r_{p}}$ and let $\hat{\theta}_{p}$ be a unit vector in the direction of $\frac{\partial}{\partial \theta_{p}}$, i.e., $\hat{\theta}_{p}=\frac{1}{r_{p}} \theta_{p}$. For $\left\{e_{1}, \ldots, e_{m}\right\}$ the standard basis of $\mathbf{R}^{m}$, define

$$
a_{i p}^{s}(x, r)=\varphi_{s}\left(\|x\|^{2},\|r\|^{2}\right)\left\langle\left[x, e_{i}\right], Z_{p}\right\rangle .
$$

Set

$$
\hat{x}_{i}^{s}=e_{i}+a_{i 1}^{s}(x, r) \frac{\partial}{\partial \theta_{1}}+\cdots+a_{i k}^{s}(x, r) \frac{\partial}{\partial \theta_{k}}=e_{i}+a_{i 1}^{s}(x, r) r_{1} \hat{\theta}_{1}+\cdots+a_{i k}^{s}(x, r) r_{k} \hat{\theta}_{k} .
$$

Then

$$
\left\{E_{1}^{s}, \ldots, E_{m+2 k}^{s}\right\}=\left\{\hat{x}_{1}^{s}, \ldots, \hat{x}_{m}^{s}, \hat{r}_{1}, \ldots, \hat{r}_{k}, \hat{\theta}_{1}, \ldots, \hat{\theta}_{k}\right\}
$$

is an orthonormal frame field on $\left(\mathbf{R}^{m+2 k}, g^{s}\right)$. (Note that $E_{\alpha}^{s}$ depends trivially on $s$ when $\alpha>m$.) Set

$$
\begin{gathered}
I_{1}=\{1, \ldots, m\}, \\
I_{2}=\{m+1, \ldots, m+k\},
\end{gathered}
$$

and

$$
I_{3}=\{m+k+1 \ldots, m+2 k\} .
$$

Lemma 5.7. We use the notation of Notation 5.6 and Definition 5.3. Let $c_{\alpha \beta}^{(s) \gamma}$ denote the structure constants given by $\left[E_{\alpha}^{s}, E_{\beta}^{s}\right]=\sum_{\gamma} c_{\alpha \beta}^{(s) \gamma} E_{\gamma}^{s}$. Then:

(i) If $\gamma \in I_{3}$ and $\alpha, \beta \in I_{1}$, then $\left\{c_{\alpha \beta}^{(s) \gamma}\right\}$ is a homogeneous $(r, s)$-deformation of degree -1 .

(ii) If $\gamma \in I_{3}$, one of $\alpha, \beta$ is in $I_{1}$ and the other is in $I_{2}$, then $\left\{c_{\alpha \beta}^{(s) \gamma}\right\}$ is a homogeneous $(r, s)$-deformation of degree 0 .

(iii) In all other cases $c_{\alpha \beta}^{(s) \gamma}=0$.

Proof. By Notation 5.6, we have for $1 \leq i, j \leq m$ and $1 \leq p \leq k$,

$$
\begin{aligned}
{\left[\hat{x}_{i}, \hat{x}_{j}\right] } & =\sum_{q=1}^{k}\left(\frac{\partial}{\partial x_{i}} a_{j q}^{s}-\frac{\partial}{\partial x_{j}} a_{i q}^{s}\right) \frac{\partial}{\partial \theta_{q}} \\
& =\sum_{q=1}^{k}\left(\frac{\partial}{\partial x_{i}} a_{j q}^{s}-\frac{\partial}{\partial x_{j}} a_{i q}^{s}\right) r_{q} \hat{\theta}_{q} \\
{\left[\hat{r}_{p}, \hat{x}_{i}\right] } & =\sum_{q=1}^{k} \frac{\partial}{\partial r_{p}} a_{i q}^{s} \frac{\partial}{\partial \theta_{q}} \\
& =\sum_{q=1}^{k} \frac{\partial}{\partial r_{p}} a_{i q}^{s} r_{q} \hat{\theta}_{q} .
\end{aligned}
$$


All other brackets of vectors in our orthonormal frame are zero. The lemma now follows from Lemma 5.4 and the fact that for each $i, p,\left\{a_{i p}^{s}\right\}$ is a homogeneous $(r, s)$ deformation of degree 0 .

Lemma 5.8. Let $\Gamma_{\alpha \beta}^{(s) \gamma}, \alpha, \beta, \gamma=1, \ldots, m+2 k$ denote the Christoffel symbols for the metric $g^{s}$ with respect to the frame field $\left\{E_{1}^{s}, \ldots, E_{m+2 k}^{s}\right\}$, i.e., $\nabla_{E_{\beta}^{s}}^{s} E_{\alpha}^{s}=\sum_{\gamma} \Gamma_{\alpha \beta}^{(s) \gamma} E_{\gamma}^{s}$, where $\nabla^{s}$ is the Levi-Civita connection for $g^{s}$. Then:

(i) If two of the indices $\alpha, \beta, \gamma$ lie in $I_{1}$ and the other lies in $I_{3}$, then $\left\{\Gamma_{\alpha \beta}^{(s) \gamma}\right\}$ is a homogeneous $(r, s)$-deformation of degree -1 .

(ii) If one of the indices $\alpha, \beta, \gamma$ lies in $I_{1}$, one lies in $I_{2}$ and one lies in $I_{3}$, then $\left\{\Gamma_{\alpha \beta}^{(s) \gamma}\right\}$ is a homogeneous $(r, s)$-deformation of degree 0 .

(iii) If the indices $\alpha, \beta, \gamma$ do not satisfy the conditions of either (i) or (ii), then $\Gamma_{\alpha \beta}^{(s) \gamma}=0$.

Thus in every case, $\left\{\Gamma_{\alpha \beta}^{(s) \gamma}\right\}$ is a homogeneous $(r, s)$-deformation. Moreover:

(iv) If $\left\{\Gamma_{\alpha \beta}^{(s) \gamma}\right\}$ is a homogeneous $(r, s)$-deformation of degree $d$, then $\left\{\Gamma_{\alpha \beta, \delta}^{(s) \gamma}\right\}$ given by $\Gamma_{\alpha \beta, \delta}^{(s) \gamma}:=E_{\delta}\left(\Gamma_{\alpha \beta}^{(s) \gamma}\right)$ is a (possibly zero) homogeneous $(r, s)$-deformation. It is of degree $d+1$ if $\delta \in I_{2}$ and of degree $d$ otherwise.

Proof. We have

$$
\begin{aligned}
\Gamma_{\alpha \beta}^{(s) \gamma} & =\frac{1}{2}\left\{g^{s}\left(\left[E_{\beta}, E_{\alpha}\right], E_{\gamma}\right)+g^{s}\left(\left[E_{\gamma}, E_{\alpha}\right], E_{\beta}\right)+g^{s}\left(\left[E_{\gamma}, E_{\beta}\right], E_{\alpha}\right)\right\} \\
& =\frac{1}{2}\left(c_{\beta \alpha}^{(s) \gamma}+c_{\gamma \alpha}^{(s) \beta}+c_{\gamma \beta}^{(s) \alpha}\right) .
\end{aligned}
$$

Thus this lemma follows from Lemma 5.7.

Proposition 5.9. Let $R_{\alpha \beta \gamma \delta}^{(s)}=g^{s}\left(R^{(s)}\left(E_{\gamma}^{s}, E_{\delta}^{s}\right) E_{\beta}^{s}, E_{\alpha}^{s}\right)$. Then $\left\{R_{\alpha \beta \gamma \delta}^{(s)}\right\}$ is an $(r, s)$ deformation of degree at most one. Moreover, if $\left\{R_{\alpha \beta \gamma \delta}^{(s)}\right\}$ has degree one, then one of the indices $\alpha, \beta, \gamma, \delta$ lies in $I_{1}$, two (including at least one of $\gamma, \delta$ ) lie in $I_{2}$ and one lies in $I_{3}$.

Proof. We have

$$
R_{\alpha \beta \gamma \delta}^{(s)}=\Gamma_{\mu \gamma}^{(s) \alpha} \Gamma_{\beta \delta}^{(s) \mu}-\Gamma_{\mu \delta}^{(s) \alpha} \Gamma_{\beta \gamma}^{(s) \mu}-c_{\gamma \delta}^{(s) \mu} \Gamma_{\beta \mu}^{(s) \alpha}+\Gamma_{\beta \delta, \gamma}^{(s) \alpha}-\Gamma_{\beta \gamma, \delta}^{(s) \alpha} .
$$

By Lemmas 5.7 and 5.8, each of the first three terms belong to $(r, s)$-deformations of non-positive degree. Also by Lemma 5.8, $\left\{\Gamma_{\beta \delta, \gamma}^{(s) \alpha}\right\}$ is a homogeneous $(r, s)$ deformation. Its degree is one if $\gamma \in I_{2}$ and if $\Gamma_{\beta \delta}^{(s) \alpha}$ has degree zero; otherwise its degree is non-positive. Again applying Lemma 5.8, if $\Gamma_{\beta \delta}^{(s) \alpha}$ has degree zero and is non-trivial, then each of $I_{1}, I_{2}$ and $I_{3}$ contains exactly one of $\alpha, \beta$, and $\delta$. The proposition now follows.

Proof of Theorem 5.2: It follows from Proposition 5.9, that the integrand $\left\{5 \tau^{2}-\right.$ $\left.\left\|\operatorname{Ric}^{(s)}\right\|^{2}+\left\|R^{(s)}\right\|^{2}\right\}$ in $\left\{a_{2}\left(g^{s}\right)\right\}$ is an $(r, s)$-deformation of degree at most 2 . We now show that it in fact has degree 2 . We have

$$
\operatorname{Ric}_{\alpha \beta}^{(s)}=\sum_{\gamma} R_{\alpha \gamma \beta \gamma}^{(s)}
$$


and

$$
\left\|\operatorname{Ric}^{(s)}\right\|^{2}=\sum_{\alpha, \beta}\left|\operatorname{Ric}_{\alpha \beta}^{(s)}\right|^{2}
$$

By Proposition 5.9, $\left\{\operatorname{Ric}_{\alpha \beta}^{(s)}\right\}$ is an $(r, s)$-deformation of degree at most one. Moreover, $\left\{\operatorname{Ric}_{\alpha \beta}^{(s)}\right\}$ has degree one only when one of $\alpha, \beta$ lies in $I_{1}$ and the other in $I_{3}$. In this case, the homogeneous term of degree one in $\operatorname{Ric}_{\alpha \beta}^{(s)}$ is equal to the homogeneous term of degree one in $R_{\alpha(m+1) \beta(m+1)}^{(s)}+\cdots+R_{\alpha(m+k) \beta(m+k)}^{(s)}$. Consequently, $\left\{\left\|\operatorname{Ric}^{(s)}\right\|^{2}\right\}$ is an $(r, s)$-deformation of degree at most two, and the homogeneous term of degree two equals

$$
2 \sum_{\alpha \in I_{1}} \sum_{\beta \in I_{3}}\left|h_{1}\left(R_{\alpha(m+1) \beta(m+1)}^{(s)}\right)+\cdots+h_{1}\left(R_{\alpha(m+k) \beta(m+k)}^{(s)}\right)\right|^{2},
$$

where we are using the notation $h_{1}(\cdot)$ to denote the homogeneous term of degree one. (The coefficient 2 is due to the symmetry when we interchange the roles of $\alpha$ and $\beta$.) From the identity $\left(a_{1}+\ldots a_{k}\right)^{2} \leq k\left(a_{1}^{2}+\cdots+a_{k}^{2}\right)$, we thus see that the homogeneous term of degree 2 in $\left\|\operatorname{Ric}^{(s)}\right\|^{2}$ is no bigger than $\left\{2 k T^{s}\right\}$ where

$$
T^{s}:=\sum_{\alpha \in I_{1}} \sum_{\beta \in I_{3}}\left(\left|h_{1}\left(R_{\alpha(m+1) \beta(m+1)}^{(s)}\right)\right|^{2}+\cdots+\left|h_{1}\left(R_{\alpha(m+k) \beta(m+k)}^{(s)}\right)\right|^{2}\right) .
$$

Next consider $\left\|R^{(s)}\right\|^{2}=\sum_{\alpha, \beta, \gamma, \delta}\left|R_{\alpha \beta \gamma \delta}^{(s)}\right|^{2}$. Note that $\left\{\left\|R^{(s)}\right\|^{2}\right\}$ is also an $(r, s)$ deformation of degree at most two. The homogeneous term of degree two equals the homogeneous term of degree two in the sum of those $\left|R_{\alpha \beta \gamma \delta}^{(s)}\right|^{2}$ for which two of the indices lie in $I_{2}$, one lies in $I_{1}$ and one in $I_{3}$. Due to the symmetries of the curvature tensor, we conclude that the homogeneous term of degree two in $\left\|R^{(s)}\right\|^{2}$ is greater than or equal to $8 T(s)$.

We conclude that the homogeneous term $f^{s}$ of degree two in $\left\{-\left\|\operatorname{Ric}^{(\mathrm{s})}\right\|^{2}+\left\|R^{(s)}\right\|^{2}\right\}$ satisfies $f^{s} \geq(8-2 k) T^{s} \geq 0$. (The latter inequality uses the hypothesis that $k \leq 3$.) By Lemma 5.5 , the heat invariant $a_{2}\left(g^{s}\right)$ is a finite real linear combination of powers of $s$ with the coefficient of $s^{2-2 k}$ being greater than or equal to $\int_{\mathbf{R}^{m+2 k}} f^{1}$. (Here we are using the fact that the homogeneous term of degree two in $\left(\tau^{s}\right)^{2}$ is the square of the homogeneous term of degree one in $\tau^{s}$ and is thus nonnegative.) Thus the theorem will follow if we show that $\int_{\mathbf{R}^{m+2 k}} f^{1}>0$. Since $f^{1} \geq 0$, it thus suffices to show that $f^{1}$ is not identically zero.

Choose $\alpha, \beta, \gamma, \delta$ as follows: Choose $i \in\{1, \ldots, m\}$ and $p \in\{1, \ldots, k\}$ so that the linear transformation $\mathbf{R}^{m} \rightarrow \mathfrak{z}$ given by $x \rightarrow\left\langle\left[x, e_{i}\right], Z_{p}\right\rangle$ is not identically zero. Without loss of generality, we assume $p=1$. Let $\beta=\delta=m+2$, let $\alpha=m+k+1$ and let $\gamma=i$. Then by Notation 5.6 and the proofs of Lemma 5.7, Lemma 5.8, and Proposition 5.9, the homogeneous term of degree one in $R_{\alpha \beta \gamma \delta}^{1}$ is given by

$$
\frac{\partial^{2}}{\partial r_{2}^{2}} a_{i 1}^{1} r_{1}=4 r_{2}^{2} \phi_{22}\left(\|x\|^{2},\|r\|^{2}\right)\left\langle\left[x, e_{i}\right], Z_{1}\right\rangle r_{1}
$$

(viewed as a function on $\mathbf{R}^{m+2 k}$ depending trivially on $\theta$ ). This function cannot be identically zero since the smooth cut-off function $\varphi$ cannot be linear in either variable. It follows that $f^{1}$ is not identically zero. This completes the proof of Theorem 5.2. 


\section{Acknowledgements}

Peter Perry thanks Carolyn Gordon and David Webb for hospitality at Dartmouth College during part of the time that this work was done. Both authors thank David Webb for helpful suggestions, and Dorothee Schueth for helpful conversations and correspondence. They also acknowledge the support of the NSF under grant DMS0207125 which supported a conference on Inverse Spectral Geometry at the University of Kentucky during which some of this work was done.

We dedicate this paper to the memory of our close friend and colleague Robert Brooks, a leading researcher in spectral geometry whose wide-ranging interests and creativity in bringing to bear upon spectral geometry original insights from areas such as complex analysis, combinatorics, algebra, and probability theory were widely admired. He had a vision of mathematics as a cooperative, collaborative endeavor, and his generosity in sharing his ideas and in collaborating widely has had a profound impact upon an entire generation of spectral geometers. We dedicate this paper as a token of our gratitude for many years of rewarding friendship and fruitful collaboration.

\section{References}

[1] P. Bérard. Transplantation et isospectralité, Math. Ann. 292 (1992) 547-560.

[2] G. E. Bredon, Introduction to compact transformation groups, Pure and Applied Mathematics, Vol. 46. Academic Press, New York-London (1972) 459 pp.

[3] R. Brooks and O. Davidovich, Isoscattering on surfaces, J. Geom. Anal. 13 (2003), no. 1, 39-53.

[4] R. Brooks, R. Gornet, and P. Perry, Isoscattering Schottky manifolds, Geom. Funct. Anal. 10 (2000), no. 2, 307-326.

[5] R. Brooks and P. Perry, Isophasal scattering manifolds in two dimensions, Comm. Math. Phys. 223 (2001), no. 3, 465-474.

[6] P. Chernoff, Essential self-adjointness of powers of generators of hyperbolic equations, J. Functional Analysis 12 (1973) 401-414.

[7] T. Christiansen, Weyl asymptotics for the Laplacian on asymptotically Euclidean spaces, Amer. J. Math. 121 (1999), no. 1, 1-22.

[8] C. Gordon, Isospectral closed Riemannian manifolds which are not locally isometric: II, Geometry of the Spectrum Contemp. Math. 173 (1994) 121-131.

[9] _ Isospectral deformations of metrics on spheres, Invent. Math. 145 (2001) 317-331.

[10] C. Gordon and E. N. Wilson, Continuous families of isospectral Riemannian manifolds which are not locally isometric, J. Differential Geom. 47 (1997) 504-529.

[11] L. Guillopé and M. Zworski. Scattering asymptotics for Riemann surfaces, Ann. Math. 145 (1997), 597-660.

[12] A. Hassell and S. Zelditch, Determinants of Laplacians in exterior domains, Int. Math. Res. Not. 18 (1999) 971-1004.

[13] E. Hebey, Nonlinear analysis on manifolds: sobolev spaces and inequalities, Courant Lecture Notes 5, 2000. Providence: American Mathematical Society, 2000.

[14] R. B. Melrose, Spectral and scattering theory for the Laplacian on asymptotically Euclidian spaces, Spectral and scattering theory (Sanda, 1992), 85-130, Lecture Notes in Pure and Appl. Math. 161, Dekker, New York, 1994.

[15] _ Geometric scattering theory. Stanford Lectures. Cambridge University Press, Cambridge, 1995.

[16] F. Klopp and M. Zworski. Generic simplicity of resonances, Helv. Phys. Acta. 6 (1995) 531-538.

[17] R. Kuwabara, On the characterization of flat metrics by the spectrum, Comment. Math. Helvetici 55 (1980) 427-444.

[18] R. B. Melrose and M. Zworski, Scattering metrics and geodesic flow at infinity, Invent. Math. 124 (1996), no. 1-3, 389-436. 
[19] E. Proctor, Isospectral metrics and potentials on classical compact simple Lie groups, Michigan Math. J. 53 (2005), no. 2, 305-318.

[20] D. Robert, Asymptotique de la phase de diffusion à haute énergie pour des perturbations du second ordre du laplacien, Ann. Sci. École Norm. Sup. (4) 25 (1992), no. 2, 107-134.

[21] A. Sá Barreto and S.-H. Tang, Existence of resonances in metric scattering, Comput. Appl. Math. 17 (1998), no. 1, 3-18.

[22] D. Schueth, Continuous families of isospectral metrics on simply connected manifolds, Ann. of Math. 149 (1999) 287-308.

[23] __ Isospectral manifolds with different local geometries, J. Reine Angew. Math. 534 (2001) 41-94.

[24] Isospectral metrics on five-dimensional spheres, J. Differential Geom. 58 (2001), no. 1, $87-111$.

[25] B. Simon, Trace Ideals and their Applications. London Math. Soc. Lecture Note Ser. 35 (1979).

[26] J. Sjöstrand and M. Zworski, Complex scaling and the distribution of scattering poles, Journal of the A. M. S. 4 (1991) 729-769.

[27] T. Sunada, T. Riemannian coverings and isospectral manifolds, Ann. Math. 121 (1985) 169186.

[28] S.-H. Tang, Existence of resonances for metric scattering in even dimensions, Lett. Math. Phys. 52 (2000), no. 3, 211-223

[29] S. Zelditch, Isospectrality in the FIO category, J. Differential Geom. 35 (1992), no. 3, 689-710.

[30] __ Kuznecov sum formulae and Szegö limit formulas on manifolds, Comm. Partial Differential Equations 17 (1992) 221-260.

[31] M. Zworski, Counting scattering poles, Spectral and scattering theory (Sanda, 1992), 301-331, Lecture Notes in Pure and Appl. Math., 161, Dekker, New York, 1994.

[32] __ Resonances in physics and geometry, Notices Amer. Math. Soc. 46 (1999), no. 3, 319328.

Department of Mathematics, Dartmouth College, Hanover, New Hampshire 03755

E-mail address: Carolyn.S.Gordon@dartmouth.edu

Department of Mathematics, University of Kentucky, Lexington, Kentucky 40506-0027

E-mail address: perry@ms.uky.edu. 